\title{
ALGEBRAIC INDEPENDENCE OF RECIPROCAL SUMS OF POWERS OF CERTAIN FIBONACCI-TYPE NUMBERS
}

\author{
Peter Bundschuh, Keijo VÄÄnÄnen
}

Abstract: The Fibonacci-type numbers in the title look like $R_{n}=g_{1} \gamma_{1}^{n}+g_{2} \gamma_{2}^{n}$ and $S_{n}=h_{1} \gamma_{1}^{n}+$ $h_{2} \gamma_{2}^{n}$ for any $n \in \mathbb{Z}$, where the $g$ 's, $h$ 's, and $\gamma^{\prime}$ 's are given algebraic numbers satisfying certain natural conditions. For fixed $k \in \mathbb{Z}_{>0}$, and for fixed non-zero periodic sequences $\left(a_{h}\right),\left(b_{h}\right),\left(c_{h}\right)$ of algebraic numbers, the algebraic independence of the series

$$
\sum_{h=0}^{\infty} \frac{a_{h}}{\gamma_{1}^{k r^{h}}}, \quad \sum_{h=0}^{\infty}, \frac{b_{h}}{\left(R_{k r^{h}+\ell}\right)^{m}}, \quad \sum_{h=0}^{\infty}, \frac{c_{h}}{\left(S_{k r^{h}+\ell}\right)^{m}} \quad\left((\ell, m, r) \in \mathbb{Z} \times \mathbb{Z}_{>0} \times \mathbb{Z}_{>1}\right)
$$

is studied. Here the main tool is Mahler's method which reduces the investigation of the algebraic independence of numbers (over $\mathbb{Q}$ ) to that of functions (over the rational function field) if they satisfy certain types of functional equations.

Keywords: algebraic independence of numbers, Mahler's method, algebraic independence of functions.

\section{Introduction and main results}

Take a binary linear recurrence $\left(R_{n}\right) \in \mathbb{Z}^{\mathbb{N}_{0}}$ defined by

$$
R_{n+2}=A R_{n+1}+B R_{n} \quad(n=0,1, \ldots),
$$

where $A, B \in \mathbb{Z} \backslash\{0\}, \Delta:=A^{2}+4 B>0$, and not both of $R_{0}, R_{1} \in \mathbb{Z}$ vanishing. Consider, for $\ell \in \mathbb{Z}, k, m, r \in \mathbb{N}, r \geqslant 2$, series of the form

$$
\sum_{h=0}^{\infty} \frac{b_{h}}{\left(R_{k r^{h}+\ell}\right)^{m}}
$$

where $\left(b_{h}\right)_{h \geqslant 0}$ is a sequence of algebraic numbers, and $\sum_{h \geqslant 0}^{\prime}$ is taken over those $h \in \mathbb{N}_{0}$ for which $k r^{h}+\ell \geqslant 0$ and $R_{k r^{h}+\ell} \neq 0$.

Systematic studies on the transcendence of examples of special series of type (2), based on the arithmetic properties of solutions of certain functional equations in

2010 Mathematics Subject Classification: primary: 11J91; secondary: 11J81, 39 B32 
several variables, began with Mahler around 1930 and seriously continued in the mid-1990s with the work of Becker and Töpfer [1]. As main result, in the particular case $m=1,\left(b_{h}\right)$ periodic but not identically vanishing, and the extra condition that $\Delta$ is not a perfect square, these authors succeeded in completely characterizing when (2) is algebraic.

Shortly later, Nishioka and her school obtained comprehensive results on the arithmetic nature of series of type (2). In particular, not only transcendence but also questions on the algebraic independence of sums of type (2) were studied. For instance, in the particular situation $m=1,\left(R_{n}\right)_{n \geqslant 0}$ is not a geometric progression, $\left(b_{h}\right)$ is a linear recurrence (but without the above extra condition on $\Delta$ ), Nishioka [8] fully determined the two exceptional cases, where the sums

$$
\sum_{h=0}^{\infty} \frac{b_{h}}{R_{k r^{h}+\ell}} \quad(\ell \in \mathbb{Z})
$$

are algebraically dependent. Notice that here $k, r \in \mathbb{N}, r \geqslant 2$ are fixed. Under the same hypotheses, but the one on $\left(b_{h}\right)$ now strengthened to the effect that this sequence is periodic and not identically zero, Nishioka [9] characterized the single exceptional case, where the sums

$$
\sum_{h=0}^{\infty} \frac{b_{h}}{R_{k r^{h}+\ell}} \quad(\ell \in \mathbb{Z}, r \in \mathbb{N}, r \geqslant 2)
$$

are algebraically dependent; here only $k \in \mathbb{N}$ is fixed.

Whereas Nishioka's papers $[8,9]$ discussed right before considered only the case $m=1$ of (2), Nishioka, Tanaka and Toshimitsu [10] allowed general $m \in \mathbb{N}$ at the cost of restricting $B$ to 1 or -1 . Their main result, Theorem 1.1, reads as follows. Let $\left(b_{h}\right)$ be a non-zero periodic sequence of algebraic numbers, and assume $k \in \mathbb{N}$ to be fixed. Then the numbers

$$
\sum_{h=0}^{\infty} \frac{b_{h}}{\left(R_{k r^{h}+\ell}\right)^{m}} \quad(\ell \in \mathbb{Z}, m, r \in \mathbb{N}, r \geqslant 2)
$$

are algebraically independent except in the following case. If $R_{\ell_{0}}=0$ for some $\ell_{0} \in \mathbb{Z}$ (see the remark below) and $\left(b_{h}\right)$ is constant, then $\theta_{0}:=\sum_{h \geqslant 0}^{\prime} b_{h} / R_{k 2^{h}+\ell_{0}}$ is algebraic, and the numbers (3), except $\theta_{0}$, are algebraically independent.

Before stating our own results, we make the following remark. Assume that an integer recurrence $\left(R_{n}\right)$ satisfies $(1)$, has $\left(R_{0}, R_{1}\right) \neq(0,0), A B \neq 0, \Delta>0$ (as at the very beginning), and is not a geometric progression. Then we know that the two distinct non-zero real roots $\gamma_{1}, \gamma_{2}$ of the companion polynomial $X^{2}-A X-B$ of (1) may be assumed to satisfy $\left.\mid \gamma_{1}\right]>\max \left(1,\left|\gamma_{2}\right|\right)$. Further, with $g_{1}:=\left(R_{1}-\right.$ $\left.\gamma_{2} R_{0}\right) /\left(\gamma_{1}-\gamma_{2}\right), g_{2}:=\left(R_{1}-\gamma_{1} R_{0}\right) /\left(\gamma_{2}-\gamma_{1}\right)$, we know

$$
R_{n}=g_{1} \gamma_{1}^{n}+g_{2} \gamma_{2}^{n} \quad\left(n \in \mathbb{N}_{0}\right),
$$

where $g_{1} g_{2} \neq 0$ since $\left(R_{n}\right)$ is not a geometric progression. We may also define $R_{n}$ for all $n \in \mathbb{Z}$ by this formula. 
This simple consideration motivates our next procedure. Namely, let $\gamma_{1}$ and $\gamma_{2}$ be non-zero algebraic numbers satisfying $\left|\gamma_{1}\right|>\max \left(1,\left|\gamma_{2}\right|\right)$, and define

$$
R_{n}:=g_{1} \gamma_{1}^{n}+g_{2} \gamma_{2}^{n}, \quad S_{n}:=h_{1} \gamma_{1}^{n}+h_{2} \gamma_{2}^{n} \quad(n \in \mathbb{Z}),
$$

where $g_{1}, g_{2}, h_{1}, h_{2}$ are non-zero algebraic numbers. Moreover, let $\Omega:=\left(g_{1} h_{2}\right) /\left(g_{2} h_{1}\right)$. In particular, the special choice $\left(R_{n}\right)=\left(F_{n}\right),\left(S_{n}\right)=\left(L_{n}\right)$ with $F_{n}, L_{n}$ the ordinary Fibonacci and Lucas numbers, respectively, yields $\Omega=-1$. Next, for fixed $k \in \mathbb{N}$, we introduce for $\ell \in \mathbb{Z}, m, r \in \mathbb{N}, r \geqslant 2$ the three series

$$
Q_{r}:=\sum_{h=0}^{\infty} \frac{a_{h}}{\gamma_{1}^{k r^{h}}}, \quad R_{\ell, r, m}:=\sum_{h=0}^{\infty}{ }^{\prime} \frac{b_{h}}{\left(R_{k r^{h}+\ell}\right)^{m}}, \quad S_{\ell, r, m}:=\sum_{h=0}^{\infty} \frac{c_{h}}{\left(S_{k r^{h}+\ell}\right)^{m}},
$$

where $\left(a_{h}\right)_{h \geqslant 0},\left(b_{h}\right)_{h \geqslant 0}$ and $\left(c_{h}\right)_{h \geqslant 0}$ are non-zero periodic sequences of algebraic numbers.

In the following results, we consider three cases:

i) $\gamma_{1}$ and $\gamma_{2}$ are multiplicatively independent,

ii) the product $\gamma_{1} \gamma_{2}$ is 1 or -1 ,

iii) $\gamma_{1}= \pm \gamma^{\kappa}, \gamma_{2}= \pm \gamma^{\nu}$ with an algebraic $\gamma,|\gamma|>1$ and $\kappa, \nu \in \mathbb{Z}$ satisfying $\kappa>\max (0, \nu)$.

Of course, in the (mutually not exclusive) cases ii) and iii), $\gamma_{1}$ and $\gamma_{2}$ are multiplicatively dependent.

Theorem 1. Assume that $\Omega=\delta\left(\gamma_{1} / \gamma_{2}\right)^{\ell_{1}}$ with some $\ell_{1} \in \mathbb{Z}$ and $|\delta|=1, \delta \neq 1$. Then the numbers

$$
Q_{r}, R_{\ell, r, m}, S_{\ell, r, m} \quad\left(\ell \in \mathbb{Z}, m \in \mathbb{N}, r \in \mathbb{N} \backslash\{1\}, r \notin 2^{2 \mathbb{N}-1}\right)
$$

are algebraically independent in cases i) and ii).

We note that, in the case ii), [4, Theorem 2] gives algebraic independence of

$$
Q_{r}, R_{\ell, r, 1}, S_{\ell, r, 1} \quad(\ell \in \mathbb{Z}, r \in \mathbb{N} \backslash\{1\})
$$

except in four explicitly given special cases. Some extra condition like $r \notin 2^{2 \mathbb{N}-1}$ is needed for $m \geqslant 2$, see [2, Theorem 2 and Remark 5].

For the following corollary, we denote by $F_{\ell, r, m}$ and $L_{\ell, r, m}$ the numbers $R_{\ell, r, m}$ and $S_{\ell, r, m}$ in (4) if $R_{n}$ and $S_{n}$ are replaced by $F_{n}$ and $L_{n}$, respectively.

Corollary 1. The numbers

$$
Q_{r}, F_{\ell, r, m}, L_{\ell, r, m} \quad\left(\ell \in \mathbb{Z}, m \in \mathbb{N}, r \in \mathbb{N} \backslash\{1\}, r \notin 2^{2 \mathbb{N}-1}\right)
$$

are algebraically independent, where $Q_{r}$ is defined as in (4) with the particular $\gamma_{1}=(1+\sqrt{5}) / 2$. 
This gives a partial generalization of [3, Theorem 7] for all $r \notin 2^{2 \mathbb{N}-1}$. But note that this Theorem 7 is also valid for $r \in 2^{2 \mathbb{N}-1}$ except for $r=2$. Moreover, also here [4, Theorem 1] implies algebraic independence of

$$
Q_{r}, F_{\ell, r, 1}, L_{\ell, r, 1} \quad(\ell \in \mathbb{Z}, r \in \mathbb{N} \backslash\{1\})
$$

unless $\left(b_{h}\right)$ is constant. In this special case $F_{0,2,1}$ is algebraic, but all other numbers are algebraically independent.

Theorem 2. In the cases i) and ii), the numbers

$$
Q_{r}, R_{\ell, r, m} \quad(\ell \in \mathbb{Z}, m \in \mathbb{N}, r \in \mathbb{N} \backslash\{1\})
$$

are algebraically independent except in the following situation. In case ii) there exists an $\ell_{0} \in \mathbb{Z}$ with $R_{\ell_{0}}=0$, and the sequence $\left(b_{h}\right)$ is constant. Then $R_{\ell_{0}, 2,1}$ is algebraic but all other numbers in (6) are algebraically independent.

Clearly, Theorem 2 fully contains [10, Theorem 1.1] quoted above since the hypothesis $B= \pm 1$ there is equivalent to $\gamma_{1} \gamma_{2}= \pm 1$ describing just our case ii).

Theorem 3. Let the assumptions of Theorem 1 hold. Then, in case iii), the numbers

$Q_{r}^{*}:=\sum_{h=0}^{\infty} \frac{a_{h}}{\gamma^{k r^{h}}}, R_{\ell, r, m}, S_{\ell_{1}+\ell, r, m} \quad\left(\ell \in \mathbb{Z}, \ell \neq \ell_{0}, m \in \mathbb{N}, r \in \mathbb{N} \backslash\{1\}, r \notin 2^{2 \mathbb{N}-1}\right)$

are algebraically independent. Here $\ell_{0} \in \mathbb{Z}$ satisfies $\left|g_{1} g_{2}^{-1}\left(\gamma_{1} / \gamma_{2}\right)^{\ell_{0}}\right|=1$ if such an integer exists; otherwise the condition $\ell \neq \ell_{0}$ has to be omitted. Further, the numbers

$$
Q_{r}^{*}, R_{\ell, r, m} \quad\left(\ell \in \mathbb{Z}, \ell \neq \ell_{0}, m \in \mathbb{N}, r \in \mathbb{N} \backslash\{1\}\right)
$$

are algebraically independent.

The preceding Theorems 1 and 3 concern the case $|\Omega|=\left|\gamma_{1} / \gamma_{2}\right|^{\ell_{1}}$ for some $\ell_{1} \in \mathbb{Z}$. If this condition is not satisfied, then we obtain analogously to Theorem 2 the following result.

Theorem 4. Assume that $|\Omega| \notin\left|\gamma_{1} / \gamma_{2}\right|^{\mathbb{Z}}$. Then, in cases i) and ii), the numbers

$$
Q_{r}, R_{\ell, r, m}, S_{\ell, r, m} \quad(\ell \in \mathbb{Z}, m \in \mathbb{N}, r \in \mathbb{N} \backslash\{1\})
$$

are algebraically independent except if, in case ii), there exists an $\ell_{0} \in \mathbb{Z}$ with $R_{\ell_{0}}=0$ and $\left(b_{h}\right)_{h \geqslant 0}$ is constant (or $S_{\ell_{0}}=0$ and $\left(c_{h}\right)_{h \geqslant 0}$ is constant). In this special case, $R_{\ell_{0}, 2,1}$ (or $S_{\ell_{0}, 2,1}$, respectively) is algebraic but all other numbers in (9) are algebraically independent. In case iii), the numbers

$$
Q_{r}^{*}, R_{\ell, r, m}, S_{\ell, r, m} \quad(\ell \in \mathbb{Z}, m \in \mathbb{N}, r \in \mathbb{N} \backslash\{1\})
$$

are algebraically independent except if there exists an $\ell_{0} \in \mathbb{Z}$ such that $\left|g_{1} g_{2}^{-1}\left(\gamma_{1} / \gamma_{2}\right)^{\ell_{0}}\right|=1$ or $\left|h_{1} h_{2}^{-1}\left(\gamma_{1} / \gamma_{2}\right)^{\ell_{0}}\right|=1$. In such a case, one has to omit all numbers $R_{\ell_{0}, r, m}$ (or $S_{\ell_{0}, r, m}$, respectively) with $r \geqslant 2, m \geqslant 1$ from (10), and then the remaining numbers are algebraically independent. 
To compare our results briefly with previous results in the literature, it appears that numbers of the type $Q_{r}$ are never included in the statements. Also the occurrence of two moderately interrelated types of series (2), namely $R_{\ell, r, m}$ and $S_{\ell, r, m}$ in Theorems 1, 3, 4 and Corollary 1, seems to be new (apart from our results [3, Theorems 7 and 8] and [4, Theorem 2]). On the other hand, there are two papers $[5,6]$ investigating the arithmetical nature of series of the form

$$
\sum_{h=0}^{\infty}{ }^{\prime} \frac{b_{h}}{\left(R_{k r^{h}+\ell}+c\right)^{m}},
$$

generalizing (2), where $c$ is a fixed algebraic number. In [5, Theorems 1.2 and 1.3] and [6, Theorem 1.1], the authors establish, in the case $m=1$ but for various algebraic sequences $\left(b_{h}\right)$, the precise conditions for $(11)$ to be algebraic. In $[6$, Theorem 1.2] one can find a first result on algebraic independence of sums of type (11), again only for $m=1$. Clearly, this remark points in a possible direction of further research.

The main ideas of the proofs of our results are based on the works [3], [9] and [10]. First, in Section 2, we consider linear independence of Mahler-type functions related to the results, and then the algebraic independence of these functions. After that, [9, Corollary of Theorem 4] (in case i)) or [10, Lemma 2.1] (in cases ii) and iii)) can be applied to obtain algebraic independence of function values with different $r \geqslant 2$. In the final Section 5 , the connection of the numbers in our theorems with such function values implies the validity of Theorems 1-4.

\section{Linear independence of functions}

In our first lemma, we are interested in the Mahler-type functions

$$
F_{i}(z):=\sum_{h=0}^{\infty} a^{h} \frac{A_{i}\left(z^{r^{h}}\right)}{B_{i}\left(z^{r^{h}}\right)}, \quad i=0,1, \ldots, m,
$$

where $r \geqslant 2$ is an integer, $a \neq 0$ is a complex number, $A_{i}(z), B_{i}(z) \in$ $\mathbb{C}[z] \backslash\{0\}, A_{i}(0)=0, B_{0}(z) \equiv 1$, and, for any $i \geqslant 1, A_{i}(z)$ and $B_{i}(z)$ are coprime, and the $B_{i}(z)$ are distinct, non-constant, and monic. Clearly

$$
a F_{i}\left(z^{r}\right)=F_{i}(z)-\frac{A_{i}(z)}{B_{i}(z)}, \quad i=0,1, \ldots, m .
$$

Lemma 1. Assume that $t_{1}, \ldots, t_{m}$ and $u$ are positive integers. For each $i=$ $1, \ldots, m$, let $B_{i}(z)$ be a polynomial having exactly $t_{i}$ distinct zeros and assume that all these zeros have the same absolute value $\omega_{i}>0$. Assume further that the polynomial $B_{m+1}(z)$ has exactly $u$ distinct zeros all having absolute value 1 . If $\omega_{i} \neq 1$ for all $i=1, \ldots, m, \omega_{i} \neq \omega_{j}$ for $i \neq j$ and $(r-1) t_{i} \geqslant t_{j}$ for all $i, j$, then

$$
g(z):=c_{0} F_{0}(z)+\cdots+c_{m} F_{m}(z)+c_{m+1} F_{m+1}(z)
$$

with $\left(c_{0}, \ldots, c_{m+1}\right) \neq \underline{0}$ is rational if and only if $c_{1}=\cdots=c_{m}=0$ and $g_{1}(z):=$ $c_{0} F_{0}(z)+c_{m+1} F_{m+1}(z)$ is rational. 
Proof. The function $g(z)$ satisfies the functional equation

$$
a g\left(z^{r}\right)=g(z)-c_{0} A_{0}(z)-c_{1} \frac{A_{1}(z)}{B_{1}(z)}-\cdots-c_{m} \frac{A_{m}(z)}{B_{m}(z)}-c_{m+1} \frac{A_{m+1}(z)}{B_{m+1}(z)} .
$$

We assume that $g(z)$ is a rational function, say $g(z)=P(z) / Q(z)$, with coprime polynomials $P(z)$ and $Q(z)$. If $\left(c_{1}, \ldots, c_{m}\right) \neq \underline{0}$, then $g(z)$ has a pole of absolute value $\neq 1$. Assuming that there exists a pole of absolute value $>1$, let $p$ be such a pole with maximal absolute value. Then $|p|=\omega_{i}$ with some $1 \leqslant i \leqslant m$. Now $p$ is not a pole of $g\left(z^{r}\right)$, so the assumptions of our lemma together with (12) imply that all $t_{i}$ distinct zeros of $B_{i}(z)$, say $\alpha_{i, \nu}\left(\nu=1, \ldots, t_{i}\right)$, are poles of $g(z)$, and therefore $r t_{i}$ numbers $\sqrt[r]{\alpha_{i, \nu}}$ are poles of $g\left(z^{r}\right)$. By the assumption $\omega_{i} \neq \omega_{j}$ for $i \neq j$, the function $a g\left(z^{r}\right)-g(z)$ has exactly $t_{j}$ poles of the same absolute value $\omega_{j}$ (if $c_{j} \neq 0$ ), and therefore at least $r t_{i}-t_{j} \geqslant t_{i}$ of the above $\sqrt[r]{\alpha_{i, \nu}}$ are poles of $g(z)$ (assuming $\sqrt[r]{\omega_{i}}=\omega_{j}$ ). Let these be $q_{1}, \ldots, q_{v}$ with $v \geqslant t_{i}$. The $r v$ numbers $\sqrt[r]{q_{i}}$ are poles of $g\left(z^{r}\right)$, and again at least $r v-t_{k} \geqslant t_{i}$ of these are poles of $g(z)$. By repeating this, we get a contradiction. The same argument works also if $g(z)$ has a pole of absolute value $<1$. Therefore $\left(c_{1}, \ldots, c_{m}\right)=\underline{0}$ proving our Lemma 1.

We now begin to consider the special class of the above functions

$$
\begin{aligned}
\gamma_{\mu}(a, z) & :=\sum_{h=0}^{\infty} a^{h} z^{\mu r^{h}} \quad(\mu=1, \ldots, r-1), \\
\varphi_{\ell, m}(a, z) & :=\sum_{h=0}^{\infty} a^{h}\left(\frac{z^{r^{h}}}{z^{2 r^{h}}-\alpha_{\ell}}\right)^{m} \quad(\ell \in \mathbb{Z}, m \geqslant 1),
\end{aligned}
$$

where $a$ and all $\alpha_{\ell}$ are non-zero complex numbers. The functions $\gamma_{\mu}(a, z)$ and $\varphi_{\ell, m}(a, z)$ satisfy the functional equations

$$
a \gamma_{\mu}\left(a, z^{r}\right)=\gamma_{\mu}(a, z)-z^{\mu}, \quad a \varphi_{\ell, m}\left(a, z^{r}\right)=\varphi_{\ell, m}(a, z)-\left(\frac{z}{z^{2}-\alpha_{\ell}}\right)^{m} .
$$

Lemma 2. Let $L$ and $M$ be positive integers. Assume that $\left|\alpha_{0}\right|=1$, and $\left|\alpha_{\ell_{1}}\right| \neq\left|\alpha_{\ell_{2}}\right|$ for $\ell_{1} \neq \ell_{2}$. If $c_{\mu}, c_{\ell, m}$ are complex numbers, not all zero, then the function

$$
\sum_{\mu=1}^{r-1} c_{\mu} \gamma_{\mu}(a, z)+\sum_{\ell=-L}^{L} \sum_{m=1}^{M} c_{\ell, m} \varphi_{\ell, m}(a, z)
$$

is rational if and only if $c_{\ell, m}=0$ for all $\ell \neq 0, m=1, \ldots, M$, and the function

$$
\sum_{\mu=1}^{r-1} c_{\mu} \gamma_{\mu}(a, z)+\sum_{m=1}^{M} c_{0, m} \varphi_{0, m}(a, z)
$$

is rational. 
Proof. If $g(z)$ denotes the function (16), then

$$
a g\left(z^{r}\right)=g(z)-A(z)-\sum_{\ell=-L}^{L} \sum_{m=1}^{M} c_{\ell, m}\left(\frac{z}{z^{2}-\alpha_{\ell}}\right)^{m},
$$

where $A(z):=c_{1} z+\cdots+c_{r-1} z^{r-1}$. We may now use Lemma 1 with $t_{\ell}=2$ for all $\ell$ having some non-zero $c_{\ell, m}$, which gives $c_{\ell, m}=0$ for all $\ell \neq 0, m=1, \ldots, M$. This proves Lemma 2.

Lemma 3. If $r \geqslant 3$, then the function (17) is not rational. If $r=2$ and $a \notin 2^{2 \mathbb{N}}$, then the function (17) is not rational except in the case $a=1, \alpha_{0}=1$. In that special case, $\varphi_{0,1}(1, z)$ is rational, but the functions $\gamma_{1}(1, z)$ and $\varphi_{0, m}(1, z)(m=$ $2, \ldots, M)$ are linearly independent over $\mathbb{C}$ modulo $\mathbb{C}(z)$.

Proof. Let now $g(z)$ denote the function (17), where at least one of the coefficients $c_{\mu}, c_{0, m}$ is non-zero, and assume $g(z) \in \mathbb{C}(z)$. Then

$$
a g\left(z^{r}\right)=g(z)-A(z)-\sum_{m=1}^{M} c_{0, m}\left(\frac{z}{z^{2}-\alpha_{0}}\right)^{m},
$$

where $A(z)$ is the same polynomial as in the proof of Lemma 2. If all $c_{0, m}=0$, then $A(z) \neq 0$ and we have a contradiction. So let $m=M_{0}$ be the greatest $m$ such that $c_{0, m} \neq 0$. Clearly, all poles $\omega$ of $g(z)$ satisfy $|\omega|=1$. Further, if $t$ is the number of distinct poles, then $t r \leqslant t+2$ or $t \leqslant 2 /(r-1)$. Thus we get a contradiction if $r \geqslant 4$.

In the case $r=3, g(z)$ may have one pole $\omega$. Then $g\left(z^{3}\right)$ has poles with arguments $\tau / 3, \tau / 3+2 \pi / 3, \tau / 3+4 \pi / 3$, where $0 \leqslant \tau=\arg \omega<2 \pi$. The righthand side of (18) has possible poles with arguments $\tau, \phi / 2, \phi / 2+\pi$, where $0 \leqslant \phi=$ $\arg \alpha_{0}<2 \pi$, a contradiction.

Next let $r=2, \alpha_{0}=1$. If $g(z)$ has a pole $\omega \neq 1$, take such a pole with minimal $\arg \omega=\tau, 0<\tau<2 \pi$. Then $\tau / 2=\pi$, a contradiction. Thus the only possible pole of $g(z)$ is $z=1$ and $g(z)=P(z) /(z-1)^{N}, P(1) \neq 0$. Now (18) implies $N=M_{0}$ and

$$
a P\left(z^{2}\right)=P(z)(z+1)^{N}-A(z)\left(z^{2}-1\right)^{N}-\sum_{m=1}^{N} c_{0, m} z^{m}\left(z^{2}-1\right)^{N-m} .
$$

This gives

$$
a P(1)=P(1) 2^{N}-c_{0, N}, \quad a P(1)=-(-1)^{N} c_{0, N} .
$$

Since $P(1) \neq 0$, we get $a\left(1-(-1)^{N}\right)=2^{N}$. If $N$ is even, we have a contradiction. If $N$ is odd, we get $a=2^{N-1}$. By our assumption $a \notin 2^{2 \mathbb{N}}$, we have a contradiction if $N>1$. In the case $N=1$, we get $a=1$, and we know that $\varphi_{0,1}(1, z) \in \mathbb{C}(z)$, see [8, Lemma 4]. Omitting $\varphi_{0,1}(1, z)$ from $g(z)$ in this special case, then $N>1$, and we have a contradiction as we saw just before. 
Let then $r=2, \alpha_{0} \neq 1$. Then $0<\arg \alpha_{0}=\phi<2 \pi$. As noticed before, the number of distinct poles of $g(z)$ is $\leqslant 2$. By comparing the poles on both sides of (18), we see that $g(z)$ must have two distinct poles, say $\omega_{1}, \omega_{2}$, with $0 \leqslant \arg \omega_{1}=\tau_{1}<\arg \omega_{2}=\tau_{2}<2 \pi$. Now the comparision of poles on both sides of (18) gives only the one possibility $\tau_{1}=0, \tau_{2}=\phi=\pi$. This leaves us with the functional equation

$$
a g\left(z^{2}\right)=g(z)-A(z)-\sum_{m=1}^{M_{0}} c_{0, m}\left(\frac{z}{z^{2}+1}\right)^{m}
$$

where

$$
g(z)=\frac{P(z)}{(z-1)^{N}(z+1)^{T}}, \quad P( \pm 1) \neq 0 .
$$

Thus $N=T=M_{0}$ and

$$
a P\left(z^{2}\right)=P(z)\left(z^{2}+1\right)^{N}-A(z)\left(z^{4}-1\right)^{N}-\left(z^{2}-1\right)^{N} \sum_{m=1}^{N} c_{0, m} z^{m}\left(z^{2}+1\right)^{N-m},
$$

implying $a P(1)=P(1) 2^{N}, a P(1)=P(-1) 2^{N}, a P(-1)=-c_{0, N} i^{N}(-2)^{N}$, $a P(-1)=-c_{0, N}(-i)^{N}(-2)^{N}$. Thus, $a=2^{N}, P(1)=P(-1)$ and, by the last two equations, $N$ is even, which is impossible since we assumed $a \notin 2^{2 \mathbb{N}}$.

We next study dependence relations of the functions (13), (14) and

$$
\lambda_{\ell, m}(a, z)=\sum_{h=0}^{\infty} a^{h}\left(\frac{z^{r^{h}}}{z^{2 r^{h}}-\beta_{\ell}}\right)^{m} \quad(\ell \in \mathbb{Z}, m \geqslant 1),
$$

which again satisfy

$$
a \lambda_{\ell, m}\left(a, z^{r}\right)=\lambda_{\ell, m}(a, z)-\left(\frac{z}{z^{2}-\beta_{\ell}}\right)^{m} .
$$

If $\left|\alpha_{\ell_{1}}\right| \neq\left|\beta_{\ell_{2}}\right|$ for $\ell_{1}, \ell_{2}$, we can apply Lemmas 2 and 3 . In the case $\left|\alpha_{\ell}\right|=$ $\left|\beta_{\ell}\right|, \alpha_{\ell} \neq \beta_{\ell}$, we prove the following results.

Lemma 4. Let $r \geqslant 3$, and assume that $L, M$ and $\alpha_{\ell}$ are as in Lemma $2, \beta_{\ell}=$ $\delta_{\ell} \alpha_{\ell},\left|\delta_{\ell}\right|=1, \delta_{\ell} \neq 1$ for all $\ell \in \mathbb{Z}$. If $c_{\mu}, c_{\ell, m}, d_{\ell, m}$ are complex numbers, not all zero, then the function

$$
\sum_{\mu=1}^{r-1} c_{\mu} \gamma_{\mu}(a, z)+\sum_{\ell=-L}^{L} \sum_{m=1}^{M} c_{\ell, m} \varphi_{\ell, m}(a, z)+\sum_{\ell=-L}^{L} \sum_{m=1}^{M} d_{\ell, m} \lambda_{\ell, m}(a, z)
$$

is rational if and only if $c_{\ell, m}=0, d_{\ell, m}=0$ for all $\ell \neq 0, m=1, \ldots, M$, and the following sum is rational:

$$
\sum_{\mu=1}^{r-1} c_{\mu} \gamma_{\mu}(a, z)+\sum_{m=1}^{M} c_{0, m} \varphi_{0, m}(a, z)+\sum_{m=1}^{M} d_{0, m} \lambda_{0, m}(a, z) .
$$


Proof. Assume that $(21)$ is rational, and let $g(z)$ denote this sum. Then

$$
a g\left(z^{r}\right)=g(z)-A(z)-\sum_{\ell=-L}^{L} \sum_{m=1}^{M} c_{\ell, m}\left(\frac{z}{z^{2}-\alpha_{\ell}}\right)^{m}-\sum_{\ell=-L}^{L} \sum_{m=1}^{M} d_{\ell, m}\left(\frac{z}{z^{2}-\beta_{\ell}}\right)^{m}
$$

where $A(z)=c_{1} z+\cdots+c_{r-1} z^{r-1}$. If, for all $\ell \neq 0$, we have either $c_{\ell, m}=0$ or $d_{\ell, m}=0,(m=1, \ldots, M)$, then the claim follows as in Lemma 2. Assume now that, for some $\ell \neq 0$, there exist non-zero numbers $c_{\ell, M_{1}}, d_{\ell, M_{2}}$, and take here $M_{1}, M_{2}$ maximal with this property. Then

$$
\sum_{m=1}^{M_{1}} c_{\ell, m}\left(\frac{z}{z^{2}-\alpha_{\ell}}\right)^{m}+\sum_{m=1}^{M_{2}} d_{\ell, m}\left(\frac{z}{z^{2}-\beta_{\ell}}\right)^{m}=\frac{P(z)}{\left(z^{2}-\alpha_{\ell}\right)^{M_{1}}\left(z^{2}-\beta_{\ell}\right)^{M_{2}}},
$$

where $P(z) \neq 0$ is a polynomial prime to $\left(z^{2}-\alpha_{\ell}\right)\left(z^{2}-\beta_{\ell}\right)$. Thus, we may apply Lemma 1 with $t_{\ell}=2$ or 4 for all $\ell$ having non-trivial $c_{\ell, m}, d_{\ell, m}$, which gives a contradiction. Thus, $c_{\ell, m}=d_{\ell, m}=0$ for all $\ell \neq 0, m=1, \ldots, M$, proving Lemma 4.

Lemma 5. Let the assumptions of Lemma 4 hold and assume $a \notin \pm 3^{\mathbb{N}}$. Then the sum (22) is not rational.

Proof. If $g(z)$ denotes the function (22), then

$$
a g\left(z^{r}\right)=g(z)-A(z)-\sum_{m=1}^{M} c_{0, m}\left(\frac{z}{z^{2}-\alpha_{0}}\right)^{m}-\sum_{m=1}^{M} d_{0, m}\left(\frac{z}{z^{2}-\beta_{0}}\right)^{m} .
$$

By virtue of Lemma 3, we may suppose that at least one of $c_{0, m}, m=1, \ldots, M$, and similarly, at least one of $d_{0, m}, m=1, \ldots, M$, does not vanish, and let $c_{0, M_{1}}, d_{0, M_{2}}$ have maximal $m$-values with this property.

This time, we get the upper bound $t \leqslant 4 /(r-1)$ for the number of distinct poles of $g(z)$. Thus, we need to consider only the values $r=3,4,5$ and $t \leqslant 2$ if $r=3$, and $t=1$ otherwise. By comparing the arguments of the possible poles on both sides of (23), we immediately get a contradiction if $r=5$.

Assume now $r=4$, and let $\omega$ denote the pole of $g(z)$ with $0 \leqslant \arg \omega=\tau<2 \pi$. The arguments of the poles on the left-hand side of $(23)$ are $\tau / 4+j \pi / 2, j=0, \ldots, 3$, and the arguments of the possible poles on the right-hand side are $\tau, \phi_{1} / 2, \phi_{1} / 2+$ $\pi, \phi_{2} / 2, \phi_{2}+\pi$, where $\phi_{1}=\min \left(\arg \alpha_{0}, \arg \beta_{0}\right), \phi_{2}=\max \left(\arg \alpha_{0}, \arg \beta_{0}\right), 0 \leqslant$ $\phi_{1}<\phi_{2}<2 \pi$. This is only possible if $\tau=2 \phi_{1}, \phi_{2}=\phi_{1}+\pi$ and $\tau=\tau / 4+j \pi / 2$ with some $j=0, \ldots, 3$. Thus, we have three possibilities, $\tau=0,2 \pi / 3$, or $4 \pi / 3$. Without loss of generality, we may assume $\arg \alpha_{0}=\phi_{1}$. To simplify the notations, let us denote $\alpha_{0}=\alpha, c_{0, m}=c_{m}, d_{0, m}=d_{m}$. Since $\omega=\alpha^{2},(23)$ is now of the form

$$
a \frac{P\left(z^{4}\right)}{\left(z^{4}-\alpha^{2}\right)^{N}}=\frac{P(z)}{\left(z-\alpha^{2}\right)^{N}}-A(z)-\sum_{m=1}^{M_{1}} c_{m}\left(\frac{z}{z^{2}-\alpha}\right)^{m}-\sum_{m=1}^{M_{2}} d_{m}\left(\frac{z}{z^{2}+\alpha}\right)^{m}
$$


with $P\left(\alpha^{2}\right) \neq 0$. In the cases $\phi_{1}=0,2 \pi / 3$, we have $\alpha^{3}=1$ giving $z^{2}-\alpha=$ $\left(z-\alpha^{2}\right)\left(z+\alpha^{2}\right)$, and in the case $\phi_{1}=\pi / 3, \alpha^{3}=-1, z^{2}+\alpha=\left(z-\alpha^{2}\right)\left(z+\alpha^{2}\right)$. Therefore, by comparing the orders of poles above, we get $M_{1}=M_{2}=N$ in all three cases. If $\phi_{1}=0$ or $2 \pi / 3$, then (24) implies

$$
\begin{aligned}
a P\left(z^{4}\right)= & P(z)\left(\left(z+\alpha^{2}\right)\left(z^{2}+\alpha\right)\right)^{N}-A(z)\left(z^{4}-\alpha^{2}\right)^{N} \\
& -\left(z^{2}+\alpha\right)^{N} \sum_{m=1}^{N} c_{m} z^{m}\left(z^{2}-\alpha\right)^{N-m}-\left(z^{2}-\alpha\right)^{N} \sum_{m=1}^{N} d_{m} z^{m}\left(z^{2}+\alpha\right)^{N-m} .
\end{aligned}
$$

At $z= \pm \alpha^{2}$, we get

$$
a P\left(\alpha^{2}\right)=P\left(\alpha^{2}\right) 4^{N}-(2 \alpha)^{N} c_{N} \alpha^{2 N}, \quad a P\left(\alpha^{2}\right)=-(2 \alpha)^{N} c_{N} \alpha^{2 N}(-1)^{N}
$$

giving $a\left(1-(-1)^{N}\right)=4^{N}$, whence $N$ is odd. Further, let $\pm \zeta$ be the solutions of $z^{2}=-\alpha$. Then, at $z= \pm \zeta$

$$
a P\left(\alpha^{2}\right)=-(-2 \alpha)^{N} d_{N} \zeta^{N}, \quad a P\left(\alpha^{2}\right)=-(-2 \alpha)^{N} d_{N}(-\zeta)^{N} .
$$

Since $N$ is odd, we get the contradiction $a P\left(\alpha^{2}\right)=0$.

Similarly, we get a contradiction in the third case $\phi_{1}=\pi / 3$.

Assume now $r=3$. Then $t \leqslant 2$, and we immediately see that we must have $t=2$. Let $\omega_{1}$ and $\omega_{2}$ be the distinct poles of $g(z)$ with arguments $0 \leqslant \tau_{1}<$ $\tau_{2}<2 \pi$, respectively. The arguments of the poles on the left-hand side of (23) are $\tau_{1} / 3+j 2 \pi / 3, \tau_{2} / 3+j 2 \pi / 3, j=0,1,2$, and the arguments of the possible poles on the right-hand side are $\tau_{1}, \tau_{2}, \phi_{1} / 2, \phi_{1} / 2+\pi, \phi_{2} / 2, \phi_{2} / 2+\pi$, where $\phi_{1}$ and $\phi_{2}$ are as above.

If $\tau_{1}=0$, then comparision of the arguments of the poles gives us one possibility, where $\phi_{1}=2 \pi / 3, \phi_{2}=4 \pi / 3, \tau_{1}=0, \tau_{2}=\pi$. By denoting $\theta=e^{\pi i / 3}$, we get $z^{3}-1=(z-1)\left(z-\theta^{2}\right)\left(z-\theta^{4}\right), z^{3}+1=(z+1)(z-\theta)\left(z-\theta^{5}\right), z^{2}-\alpha_{0}=$ $(z-\theta)\left(z-\theta^{4}\right), z^{2}-\beta_{0}=\left(z-\theta^{2}\right)\left(z-\theta^{5}\right)$, where we assumed (without loss of generality) $\phi_{1}=\arg \alpha_{0}, \phi_{2}=\arg \beta_{0}$. Now (23) takes the shape

$$
\begin{aligned}
a \frac{P\left(z^{3}\right)}{\left(z^{3}-1\right)^{N}\left(z^{3}+1\right)^{T}}= & \frac{P(z)}{(z-1)^{N}(z+1)^{T}}-A(z)-\sum_{m=1}^{M_{1}} c_{m}\left(\frac{z}{(z-\theta)\left(z-\theta^{4}\right)}\right)^{m} \\
& -\sum_{m=1}^{M_{2}} d_{m}\left(\frac{z}{\left(z-\theta^{2}\right)\left(z-\theta^{5}\right)}\right)^{m}
\end{aligned}
$$

with $P( \pm 1) \neq 0$. By comparing the orders of poles, we obtain $N=T=M_{1}=M_{2}$, which then yields

$$
a P(1)=P(1)\left((1-\theta)\left(1-\theta^{2}\right)\left(1-\theta^{4}\right)\left(1-\theta^{5}\right)\right)^{N}=P(1) 3^{N} .
$$

Since $P(1) \neq 0$, this contradicts our assumption $a \notin 3^{\mathbb{N}}$. 
In the case $\tau_{1}>0$, we have also one possibility, $\phi_{1}=\pi / 3, \phi_{2}=5 \pi / 3, \tau_{1}=$ $\pi / 2, \tau_{2}=3 \pi / 2$. By $(23)$,

$$
\begin{aligned}
a \frac{P\left(z^{3}\right)}{\left(z^{3}-i\right)^{N}\left(z^{3}+i\right)^{T}}= & \frac{P(z)}{(z-i)^{N}(z+i)^{T}}-A(z) \\
& -\sum_{m=1}^{M_{1}} c_{m}\left(\frac{z}{z^{2}-\theta}\right)^{m}-\sum_{m=1}^{M_{2}} d_{m}\left(\frac{z}{z^{2}-\theta^{5}}\right)^{m},
\end{aligned}
$$

where $P( \pm i) \neq 0$. As before, $N=T=M_{1}=M_{2}$ and

$$
a^{2} P(i)=a P(-i) 3^{N}=P(i) 3^{2 N},
$$

which contradicts our assumption $a \notin \pm 3^{\mathbb{N}}$, whence our Lemma 5 .

We next consider the functions

$$
\begin{array}{ll}
x_{\ell, m}(a, z):=\sum_{h=0}^{\infty} a^{h}\left(\frac{z^{\kappa r^{h}}}{z^{(\kappa-\nu) r^{h}}-\alpha_{\ell}}\right)^{m} \quad(\ell \in \mathbb{Z}, m \geqslant 1), \\
y_{\ell, m}(a, z):=\sum_{h=0}^{\infty} a^{h}\left(\frac{z^{\kappa r^{h}}}{z^{(\kappa-\nu) r^{h}}-\beta_{\ell}}\right)^{m} \quad(\ell \in \mathbb{Z}, m \geqslant 1),
\end{array}
$$

where $\kappa, \nu \in \mathbb{Z}$ satisfy $\kappa>\max (0, \nu)$.

Lemma 6. If the assumptions of Lemma 4 are valid, and if $c_{\mu}, c_{\ell, m}, d_{\ell, m}$ are complex numbers, not all zero, then the function

$$
\sum_{\mu=1}^{r-1} c_{\mu} \gamma_{\mu}(a, z)+\sum_{0<|\ell| \leqslant L} \sum_{m=1}^{M} c_{\ell, m} x_{\ell, m}(a, z)+\sum_{0<|\ell| \leqslant L} \sum_{m=1}^{M} d_{\ell, m} y_{\ell, m}(a, z)
$$

is not rational. Moreover, if $r=2$ and $c_{1}, c_{\ell, m}$ are complex numbers, not all zero, then the following function is not rational:

$$
c_{1} \gamma_{1}(a, z)+\sum_{0<|\ell| \leqslant L} \sum_{m=1}^{M} c_{\ell, m} x_{\ell, m}(a, z) .
$$

Proof. The proof is a similar application of Lemma 1 as the proof of Lemma 4, but this time we have $t_{\ell}=\kappa-\nu$ or $2(\kappa-\nu)$, hence $(r-1) t_{i} \geqslant t_{j}$ for all $r \geqslant 3$. When dealing with the second claim, we have $t_{\ell}=\kappa-\nu$ for all $\ell$, and Lemma 1 applies also if $r=2$.

\section{Algebraic independence of functions}

For a sequence $\left(a_{h}\right)_{h \geqslant 0}$ of complex numbers, we denote by $\left(a_{h}^{(j)}\right)_{h \geqslant 0}, j \in \mathbb{N}$, the sequences

$$
\begin{aligned}
& \left(a_{h}^{(1)}\right)=\left(a_{0}, a_{1}, \ldots\right), \quad\left(a_{h}^{(2)}\right)=\left(a_{0}, 0, a_{1}, 0, a_{2}, 0, \ldots\right), \\
& \left(a_{h}^{(3)}\right)=\left(a_{0}, 0,0, a_{1}, 0,0, a_{2}, 0,0 \ldots\right), \quad \ldots
\end{aligned}
$$

By [10, Lemmas 2.7 and 2.8], these sequences have the following properties: 
a) If $\left(a_{h}\right)_{h \geqslant 0}$ is a periodic sequence, not identically zero, then the sequences $\left(a_{h}^{(j)}\right)_{h \geqslant 0}(j \geqslant 1)$ are linearly independent over $\mathbb{C}$.

b) If $\left(a_{h}\right)_{h \geqslant 0}$ is a periodic sequence with period length greater than 1 , then $(1),\left(a_{h}^{(j)}\right)_{h \geqslant 0}(j \geqslant 1)$ are linearly independent over $\mathbb{C},(1)$ being the obvious constant sequence.

The following lemma will be needed (see [10, p.106]).

Lemma 7. Let $p$ be a positive integer, $\left(B_{h}\right)_{h \geqslant 0}$ a periodic sequence with period length dividing $p$, and let $R(z)$ be the quotient of two polynomials in $z=\left(z_{1}, \ldots, z_{n}\right)$ such that the numerator vanishes at the origin of $\mathbb{C}^{n}$ but the denominator does not. Further, define

$$
f_{r}(z)=\sum_{h=0}^{\infty} B_{h} R\left(z^{r^{h}}\right)
$$

Then, for any $s \in \mathbb{N}$,

$$
f_{r^{j}}\left(z^{r^{s ! p}}\right)=f_{r^{j}}(z)+R_{r, j}(z)
$$

holds for $j=1, \ldots, s$ with rational functions $R_{r, j}(z)$. (Note here $\left.z^{k}=\left(z_{1}^{k}, \ldots, z_{n}^{k}\right)\right)$.

Proof. According to the definition of $f_{r}(z)$, we have $f_{r^{j}}(z)=\sum_{h \geqslant 0} B_{h} R\left(z^{r^{j h}}\right)$, hence

$$
\begin{aligned}
f_{r^{j}}\left(z^{r^{s ! p}}\right) & =\sum_{h=0}^{\infty} B_{h} R\left(z^{r^{j(h+(s ! / j) p)}}\right) \\
& =\sum_{\widetilde{h}=(s ! / j) p}^{\infty} B_{\widetilde{h}-(s ! / j) p} R\left(z^{r^{j \widetilde{h}}}\right)=\sum_{\widetilde{h}=\widetilde{h}(j)}^{\infty} B_{\widetilde{h}} R\left(z^{r^{j \widetilde{h}}}\right)
\end{aligned}
$$

(with $\widetilde{h}(j):=(s ! / j) p \in \mathbb{N})$, and this equals $f_{r^{j}}(z)$ up to addition by a rational function.

Let now $t_{1}, t_{2}, t_{3} \in \mathbb{N}$, and suppose

$$
q_{k, \chi}(z) \in \mathbb{C}\left(z_{1}, \ldots, z_{n}\right) \quad\left(k=1,2,3 ; \chi=1, \ldots, t_{k}\right),
$$

where the numerators vanish at the origin but the denominators do not. Then we define

$$
f_{k, \chi}(a, z):=\sum_{h=0}^{\infty} a^{h} q_{k, \chi}\left(z^{r^{h}}\right) \quad\left(k=1,2,3 ; \chi=1, \ldots, t_{k}\right) .
$$

Further, let $\left(b_{k, h}\right)_{h \geqslant 0}(k=1,2,3)$ be non-zero periodic sequences of complex numbers with period lengths $p_{k}$, respectively, put $p:=\operatorname{lcm}\left(p_{1}, p_{2}, p_{3}\right)$, and denote

$$
f_{k, \chi, r}(z):=\sum_{h=0}^{\infty} b_{k, h} q_{k, \chi}\left(z^{r^{h}}\right) \quad\left(k=1,2,3 ; \chi=1, \ldots, t_{k}\right) .
$$

By following ideas of [10, pp.106-107], we can now prove 
Lemma 8. If, for any root of unity $\zeta$, the functions (25) formed with $a=\zeta$ are linearly independent over $\mathbb{C}$ modulo $\mathbb{C}\left(z_{1}, \ldots, z_{n}\right)$, then the functions

$$
f_{k, \chi, r^{j}}(z) \quad\left(j \in \mathbb{N} ; k=1,2,3 ; \chi=1, \ldots, t_{k}\right)
$$

are algebraically independent over $\mathbb{C}\left(z_{1}, \ldots, z_{n}\right)$.

Proof. Assume, on the contrary, that there exists an $s \in \mathbb{N}$ such that the functions

$$
f_{k, \chi, r^{j}}(z) \quad\left(j=1, \ldots, s ; k=1,2,3 ; \chi=1, \ldots, t_{k}\right)
$$

are algebraically dependent. By Lemma 7, we may apply [7, Corollary of Theorem 3.2.1], whence these functions are linearly dependent over $\mathbb{C}$ modulo $\mathbb{C}\left(z_{1}, \ldots, z_{n}\right)$. Thus, there exist complex numbers $a_{k, \chi, j}$, not all zero, such that

$$
G(z):=\sum_{k=1}^{3} \sum_{\chi=1}^{t_{k}} \sum_{j=1}^{s} a_{k, \chi, j} f_{k, \chi, r^{j}}(z) \in \mathbb{C}\left(z_{1}, \ldots, z_{n}\right) .
$$

Since

$$
f_{k, \chi, r^{j}}(z)=\sum_{h=0}^{\infty} b_{k, h} q_{k, \chi}\left(z^{r^{j h}}\right)=\sum_{h=0}^{\infty} b_{k, h}^{(j)} q_{k, \chi}\left(z^{r^{h}}\right)
$$

we obtain

$$
G(z)=\sum_{k=1}^{3} \sum_{\chi=1}^{t_{k}} \sum_{h=0}^{\infty}\left(\sum_{j=1}^{s} a_{k, \chi, j} b_{k, h}^{(j)}\right) q_{k, \chi}\left(z^{r^{h}}\right) .
$$

Here all sequences

$$
\left(\sum_{j=1}^{s} a_{k, \chi, j} b_{k, h}^{(j)}\right)_{h \geqslant 0}
$$

are periodic with period lengths dividing $s ! p$, and therefore there exist complex numbers $A_{k, \chi, i}$ such that, for all $h \geqslant 0$,

$$
\sum_{j=1}^{s} a_{k, \chi, j} b_{k, h}^{(j)}=\sum_{i=0}^{s ! p-1} A_{k, \chi, i} \omega^{i h}
$$

with a primitive $(s ! p)$-th root of unity $\omega$. By property a) from the beginning of this section, not all $A_{k, \chi, i}$ vanish. Thus, $G(z)$ has the form

$$
G(z)=\sum_{k=1}^{3} \sum_{\chi=1}^{t_{k}} \sum_{i=0}^{s ! p-1} A_{k, \chi, i} f_{k, \chi}\left(\omega^{i}, z\right)=\sum_{i=0}^{s ! p-1} G_{i}(z) \in \mathbb{C}\left(z_{1}, \ldots, z_{n}\right)
$$

where

$$
G_{i}(z):=\sum_{k=1}^{3} \sum_{\chi=1}^{t_{k}} A_{k, \chi, i} f_{k, \chi}\left(\omega^{i}, z\right)
$$


Here

$$
\omega^{i} G_{i}\left(z^{r}\right)-G_{i}(z) \in \mathbb{C}\left(z_{1}, \ldots, z_{n}\right) .
$$

Let now $J:=\left\{i: 0 \leqslant i \leqslant s ! p-1\right.$ and at least one $\left.A_{k, \chi, i} \neq 0\right\}$. As noted above, $J$ is not empty. By applying again [7, Corollary of Theorem 3.2.1] to the functions $G_{i}(z), i \in J$, it follows that there exists an $i_{0} \in J$ such that $G_{i_{0}}(z) \in \mathbb{C}\left(z_{1}, \ldots, z_{n}\right)$ (note that the $\omega^{i}$ are distinct). By our hypothesis on the linear independence of the $f_{k, \chi}\left(\omega^{i_{0}}, z\right)$, we get the contradiction that all $A_{k, \chi, i_{0}}$ vanish, proving Lemma 8 .

For $z:=\left(z_{1}, z_{2}\right)$ we now define roots of unity $\zeta$, and $\alpha_{\ell}, \beta_{\ell}$ as in Lemma 4 ,

$$
\begin{aligned}
t_{\mu, r}(\zeta, z) & :=\sum_{h=0}^{\infty} \zeta^{h} z_{1}^{\mu r^{h}}, \quad u_{\ell, r, m}(\zeta, z):=\sum_{h=0}^{\infty} \zeta^{h}\left(\frac{z_{1}^{r^{h}}}{z_{2}^{r^{h}}-\alpha_{\ell}}\right)^{m}, \\
v_{\ell, r, m}(\zeta, z) & :=\sum_{h=0}^{\infty} \zeta^{h}\left(\frac{z_{1}^{r^{h}}}{z_{2}^{r^{h}}-\beta_{\ell}}\right)^{m} \quad(\mu=1, \ldots, r-1 ;-L \leqslant \ell \leqslant L ; m=1, \ldots, M) .
\end{aligned}
$$

Lemma 9. If $r \geqslant 3$, then the functions (29) are linearly independent over $\mathbb{C}$ modulo $\mathbb{C}\left(z_{1}, z_{2}\right)$. If $r=2$, then the same holds for the functions $t_{1,2}(\zeta, z)$ and $u_{\ell, 2, m}(\zeta, z)$.

Proof. Assume that there exist complex numbers $a_{\mu}, b_{\ell, m}, c_{\ell, m}$, not all zero, such that

$G(z):=\sum_{\mu=1}^{r-1} a_{\mu} t_{\mu, r}(\zeta, z)+\sum_{\ell=-L}^{L} \sum_{m=1}^{M}\left(b_{\ell, m} u_{\ell, r, m}(\zeta, z)+c_{\ell, m} v_{\ell, r, m}(\zeta, z)\right) \in \mathbb{C}\left(z_{1}, z_{2}\right)$.

By [7, Lemma 3.3.10], there exist polynomials $A\left(z_{1}, z_{2}\right), B\left(z_{1}, z_{2}\right) \in \mathbb{C}\left[z_{1}, z_{2}\right]$ such that

$$
G\left(z_{1}, z_{2}\right)=\frac{A\left(z_{1}, z_{2}\right)}{B\left(z_{1}, z_{2}\right)}, \quad B(0,0) \neq 0 .
$$

By choosing $z_{1}=z, z_{2}=z^{2}$, Lemmas 4 and 5 lead to a contradiction for all $r \geqslant 3$.

Let $r=2$, and assume that

$$
g(z):=a_{1} t_{1,2}(\zeta, z)+\sum_{\ell=-L}^{L} \sum_{m=1}^{M} b_{\ell, m} u_{\ell, 2, m}(\zeta, z) \in \mathbb{C}\left(z_{1}, z_{2}\right)
$$

with non-trivial complex coefficients. Again

$$
g\left(z_{1}, z_{2}\right)=\frac{A\left(z_{1}, z_{2}\right)}{B\left(z_{1}, z_{2}\right)}, \quad B(0,0) \neq 0 .
$$

Thus the choice $z_{1}=z, z_{2}=z^{2}$ leads to

$$
g\left(z, z^{2}\right)=a_{1} \gamma_{1}(\zeta, z)+\sum_{\ell=-L}^{L} \sum_{m=1}^{M} b_{\ell, m} \varphi_{\ell, m}(\zeta, z) \in \mathbb{C}(z) .
$$


By Lemma $2, b_{\ell, m}=0$ holds for all $\ell \neq 0, m=1, \ldots, M$, implying

$$
g(z)=a_{1} t_{1,2}(\zeta, z)+\sum_{m=1}^{M} b_{0, m} u_{0,2, m}(\zeta, z) \in \mathbb{C}\left(z_{1}, z_{2}\right)
$$

Now the choice $z_{1}=z_{2}=z$ gives

$$
g^{*}(z):=g(z, z) \in \mathbb{C}(z)
$$

satisfying

$$
\zeta g^{*}\left(z^{2}\right)=g^{*}(z)-a_{1} z-\sum_{m=1}^{M} b_{0, m}\left(\frac{z}{z-\alpha_{0}}\right)^{m} .
$$

Now, comparing the possible poles on both sides, we see that the only possibility is $\alpha_{0}=-1, g^{*}(z)=P(z) /(z-1)^{N}$ with $P(1) \neq 0$ and $N$ being the maximal $m \in\{1, \ldots, M\}$ satisfying $b_{0, m} \neq 0$. Thus,

$$
\zeta P\left(z^{2}\right)=P(z)(z+1)^{N}-a_{1} z\left(z^{2}-1\right)^{N}-(z-1)^{N} \sum_{m=1}^{N} b_{0, m} z^{m}(z+1)^{N-m}
$$

which gives $\zeta=2^{N}$, a contradiction. This proves Lemma 9 .

Next, let us define

$$
\begin{aligned}
t_{\mu, r}(z) & :=\sum_{h=0}^{\infty} a_{h} z_{1}^{\mu r^{h}}, \quad u_{\ell, r, m}(z):=\sum_{h=0}^{\infty} b_{h}\left(\frac{z_{1}^{r^{h}}}{z_{2}^{r^{h}}-\alpha_{\ell, r}}\right)^{m}, \\
v_{\ell, r, m}(z) & :=\sum_{h=0}^{\infty} c_{h}\left(\frac{z_{1}^{r^{h}}}{z_{2}^{r^{h}}-\beta_{\ell, r}}\right)^{m}
\end{aligned}
$$

for $\mu=1, \ldots, r-1, \ell \in \mathbb{Z}, m \in \mathbb{N}$. Then Lemmas 8 and 9 immediately give the following.

Lemma F1. Assume that $r \geqslant 3$, that $\alpha_{\ell, r}$ and $\beta_{\ell, r}$ satisfy the conditions on $\alpha_{\ell}$ and $\beta_{\ell}$ in Lemma 4 , and that $\alpha_{\ell, r^{j}}:=\alpha_{\ell, r}, \beta_{\ell, r^{j}}:=\beta_{\ell, r}$ for any $j \in \mathbb{N}$. Then the functions

$$
t_{\mu, r^{j}}(z), u_{\ell, r^{j}, m}(z), v_{\ell, r^{j}, m}(z) \quad(\mu=1, \ldots, r-1, \ell \in \mathbb{Z}, j, m \in \mathbb{N})
$$

are algebraically independent over $\mathbb{C}\left(z_{1}, z_{2}\right)$. If $r=2$, the same holds for the functions $t_{1,2^{j}}(z)$ and $u_{\ell, 2^{j}, m}(z)$.

To prepare the case $\gamma_{1} \gamma_{2}= \pm 1$, we consider the functions of one variable

$$
\gamma_{\mu, r}(z):=\sum_{h=0}^{\infty} a_{h} z^{\mu r^{h}} \quad(\mu=1, \ldots, r-1)
$$




$$
\begin{array}{ll}
\varphi_{\ell, r, m}(z):=\sum_{h=0}^{\infty} b_{h}\left(\frac{z^{r^{h}}}{z^{2 r^{h}}-\alpha_{\ell, r}}\right)^{m} \quad(\ell \in \mathbb{Z}, r, m \in \mathbb{N}, r \geqslant 2), \\
\lambda_{\ell, r, m}(z):=\sum_{h=0}^{\infty} c_{h}\left(\frac{z^{r^{h}}}{z^{2 r^{h}}-\beta_{\ell, r}}\right)^{m} \quad(\ell \in \mathbb{Z}, r, m \in \mathbb{N}, r \geqslant 2) .
\end{array}
$$

Using Lemmas 4, 5 and 8, we obtain

Lemma F2. Assume $r \geqslant 3$, and let $\alpha_{\ell, r}$ and $\beta_{\ell, r}$ satisfy the conditions of Lemma F1. Then the functions

$$
\gamma_{\mu, r^{j}}(z), \varphi_{\ell, r^{j}, m}(z), \lambda_{\ell, r^{j}, m}(z) \quad(\mu=1, \ldots, r-1, \ell \in \mathbb{Z}, j, m \in \mathbb{N})
$$

are algebraically independent over $\mathbb{C}(z)$.

Lemma F3. Assume $r \geqslant 2$, let $\alpha_{\ell, r}$ satisfy the conditions on $\alpha_{\ell}$ in Lemma 2, and let $\alpha_{\ell, r^{j}}:=\alpha_{\ell, r}$ for all $j \in \mathbb{N}$. Then the functions

$$
\gamma_{\mu, r^{j}}(z), \varphi_{\ell, r^{j}, m}(z) \quad(\mu=1, \ldots, r-1, \ell \in \mathbb{Z}, j, m \in \mathbb{N})
$$

are algebraically independent over $\mathbb{C}(z)$ except if $r=2$ and $\left(b_{h}\right)$ is constant and $\alpha_{0,2}=1$. In this special case, $\varphi_{0,2,1}(z) \in \mathbb{C}(z)$, but all other functions in (35) are algebraically independent over $\mathbb{C}(z)$.

Proof. Lemma F3 is a special case of Lemma F2 if $r \geqslant 3$. Assume now $r=2$. We may apply the proof of Lemma 8 , where $\left(b_{1, h}\right)=\left(a_{h}\right),\left(b_{2, h}\right)=\left(b_{h}\right), f_{1,1}(a, z)=$ $\gamma_{1}(a, z) \quad($ with $r=2), f_{1,1,2^{j}}(z)=\gamma_{1,2^{j}}(z)\left(t_{1}=1, j \geqslant 1\right), f_{2, \chi}(a, z)$ $\left(t_{2}=(2 L+1) M\right)$ are the functions $\varphi_{\ell, m}(a, z)(-L \leqslant \ell \leqslant L, m=1, \ldots, M)$ with $r=2$, and $f_{2, \chi, 2}(z)$ are the functions $\varphi_{\ell, 2, m}(z)(-L \leqslant \ell \leqslant L, m=1, \ldots, M)$, and all functions with $k=3$ are omitted (all $a_{3, \chi, j}=0$ ). In particular, let $\varphi_{0,1}(a, z)=$ $f_{2,1}(a, z)$ and $\varphi_{0,2,1}(z)=f_{2,1,2}(z)$. Assume also that $\varphi_{0,2,1}(z)=f_{2,1,2}(z)$ is omitted if $\left(b_{h}\right)$ is constant and $\alpha_{0,2}=1$, so $a_{2,1,1}=0$ in this case.

In the case $\alpha_{0,2} \neq 1$, we get a contradiction as in Lemma 8 by using Lemmas 2 and 3. If $\alpha_{0,2}=1$, then $f_{2,1}(1, z)=\varphi_{0,1}(1, z) \in \mathbb{C}(z)$, and thus (28) yields

$$
G(z)-A_{2,1,0} f_{2,1}(1, z)=\sum_{i=0}^{s ! p-1} G_{i}^{*}(z) \in \mathbb{C}(z)
$$

where $G_{i}^{*}(z)=G_{i}(z)$ for $i \in \mathbb{N}$ but $G_{0}^{*}(z)=G_{0}(z)-A_{2,1,0} f_{2,1}(1, z)$. We now define $J^{*}$ as $J$ in the proof of Lemma 8 , but this time $A_{2,1,0}$ is replaced by 0 in this definition (thus we may have $0 \in J$ but $0 \notin J^{*}$ ). If $J^{*}$ is not empty, we get a contradiction as in the proof of Lemma 8. Therefore we now deduce that $A_{k, m, i}=0$ for all $(k, m, i) \neq(2,1,0)$. Thus, $A_{2,1,0} \neq 0$ and, by $(27)$,

$$
\sum_{j=1}^{s} a_{2,1, j} b_{h}^{(j)}=A_{2,1,0} \quad\left(h \in \mathbb{N}_{0}\right) .
$$


If $\left(b_{h}\right)_{h \geqslant 0}$ is not constant, then the sequences $\left(b_{h}^{(j)}\right)_{h \geqslant 0}(1 \leqslant j \leqslant s)$ and $(1)_{h \geqslant 0}$ are linearly dependent contrary to property b) from the beginning of this section. If $\left(b_{h}\right)_{h \geqslant 0}$ is constant, then $a_{2,1,1}=0$, and hence $\left(b_{h}^{(j)}\right)_{h \geqslant 0}(2 \leqslant j \leqslant s)$ and $(1)_{h \geqslant 0}$ are linearly dependent contrary to property a). Thus, Lemma F3 holds.

We finally need to consider the functions

$$
\begin{aligned}
& x_{\ell, r, m}(z):=\sum_{h=0}^{\infty} b_{h}\left(\frac{z^{\kappa r^{h}}}{z^{(\kappa-\nu) r^{h}}-\alpha_{\ell, r}}\right)^{m} \quad(\ell \in \mathbb{Z}, r, m \in \mathbb{N}, r \geqslant 2), \\
& y_{\ell, r, m}(z):=\sum_{h=0}^{\infty} c_{h}\left(\frac{z^{\kappa r^{h}}}{z^{(\kappa-\nu) r^{h}}-\beta_{\ell, r}}\right)^{m} \quad(\ell \in \mathbb{Z}, r, m \in \mathbb{N}, r \geqslant 2) .
\end{aligned}
$$

By Lemmas 6 and 8, we obtain the following.

Lemma F4. Assume $r \geqslant 3$, and let $\alpha_{\ell, r}, \beta_{\ell, r}$ satisfy the conditions of Lemma F1. Then the functions

$$
\gamma_{\mu, r^{j}}(z), x_{\ell, r^{j}, m}(z), y_{\ell, r^{j}, m}(z) \quad(\mu=1, \ldots, r-1, \ell \in \mathbb{Z} \backslash\{0\}, j, m \in \mathbb{N})
$$

are algebraically independent over $\mathbb{C}(z)$. If $r=2$, the same assertion holds for the functions $\gamma_{1,2^{j}}(z), x_{\ell, 2^{j}, m}(z)$.

Remark. Note that, in [9], the author is able to remove the condition $\ell \neq 0$, if $m=1$ and the functions $\gamma_{\mu, r^{j}}(z), y_{\ell, r^{j}, 1}(z)$ are omitted.

\section{Algebraic independence of function values}

For the following consideration, along the lines of [10], we define

$$
\mathcal{R}:=\left\{r \in \mathbb{N}: r \neq a^{n} \text { with } a, n \in \mathbb{N}, n \geqslant 2\right\} .
$$

Then, according to $\left[10\right.$, p. 105], $\mathbb{N} \backslash\{1\}=\left\{r^{j}: r \in \mathcal{R}, j \in \mathbb{N}\right\}$ and $\log r_{1} / \log r_{2} \notin \mathbb{Q}$ for distinct $r_{1}, r_{2} \in \mathcal{R}$.

Let $r_{1}, \ldots, r_{t} \in \mathcal{R}, 3 \leqslant r_{1}<\cdots<r_{t}$, and let $\mathcal{R}_{1}:=\left\{r_{0}, r_{1}, \ldots, r_{t}\right\}$, where $r_{0}=2$ or $r_{0}=4$. Then $\log r_{i} / \log r_{k} \notin \mathbb{Q}$ holds for $i \neq k$. Thus, by using Lemma 7 and [10, Lemma 2.1] or [9, Corollary of Theorem 4], we may consider our functions with $r \in \mathcal{R}_{1}$.

Now, let $L, M$ and $s$ be positive integers. Assume that, for each $r \in \mathcal{R}_{1}$, we have non-zero algebraic numbers $\alpha_{\ell, r}, \beta_{\ell, r}(-L \leqslant \ell \leqslant L)$ as in Lemmas F1-F3 above. If $\alpha$ and $\beta$ are algebraic numbers with $0<|\alpha|,|\beta|<1$, then we may choose an $h_{0} \in \mathbb{N}$ in such a way that $\max (|\alpha|,|\beta|)^{2^{h_{0}}}<\min \left(\left|\alpha_{\ell, r}\right|,\left|\beta_{\ell, r}\right|:-L \leqslant \ell \leqslant L, r \in \mathcal{R}_{1}\right)$. If the functions

$$
\begin{array}{cccc}
T_{\mu, r}\left(z_{1}, z_{2}\right), & U_{\ell, r, m}\left(z_{1}, z_{2}\right), & V_{\ell, r, m}\left(z_{1}, z_{2}\right), & \Gamma_{\mu, r}(z), \\
\Phi_{\ell, r, m}(z), & \Lambda_{\ell, r, m}(z), & X_{\ell, r, m}(z), & Y_{\ell, r, m}(z)
\end{array}
$$


are defined as in (30)-(34) and (36) above but now with summation starting from $h_{0}$, then all numbers

$$
\begin{array}{cccc}
T_{\mu, r^{j}}(\alpha, \beta), & U_{\ell, r^{j}, m}(\alpha, \beta), & V_{\ell, r^{j}, m}(\alpha, \beta), & \Gamma_{\mu, r^{j}}(\alpha), \\
\Phi_{\ell, r^{j}, m}(\alpha), & \Lambda_{\ell, r^{j}, m}(\alpha), & X_{\ell, r^{j}, m}(\alpha), & Y_{\ell, r^{j}, m}(\alpha)
\end{array}
$$

with

$$
r \in \mathcal{R}_{1}, \quad 1 \leqslant \mu \leqslant r-1, \quad-L \leqslant \ell \leqslant L, \quad 1 \leqslant j \leqslant s, \quad 1 \leqslant m \leqslant M
$$

are defined. For these numbers, we can state the following results.

Theorem F1. Assume $r_{0}=4$, and let $\alpha_{\ell, r}, \beta_{\ell, r}$ satisfy the conditions of Lemma F1. Then the numbers

$$
\begin{aligned}
T_{1, r^{j}}(\alpha, \beta), \quad U_{\ell, r^{j}, m}(\alpha, \beta), \quad & V_{\ell, r^{j}, m}(\alpha, \beta) \\
& \left(r \in \mathcal{R}_{1},-L \leqslant \ell \leqslant L, 1 \leqslant j \leqslant s, 1 \leqslant m \leqslant M\right)
\end{aligned}
$$

are algebraically independent if $\alpha$ and $\beta$ are multiplicatively independent. Further, the numbers

$$
\Gamma_{\mu, r^{j}}(\alpha), \quad \Phi_{\ell, r^{j}, m}(\alpha), \quad \Lambda_{\ell, r^{j}, m}(\alpha)
$$

with (37) are algebraically independent.

Theorem F2. Let $r_{0}=2$ and $\alpha_{\ell, r}$ satisfy the conditions of Lemma F2. Then the numbers

$$
T_{1, r^{j}}(\alpha, \beta), \quad U_{\ell, r^{j}, m}(\alpha, \beta) \quad\left(r \in \mathcal{R}_{1},-L \leqslant \ell \leqslant L, 1 \leqslant j \leqslant s, 1 \leqslant m \leqslant M\right)
$$

are algebraically independent if $\alpha$ and $\beta$ are multiplicatively independent. Also the numbers

$$
\Gamma_{\mu, r^{j}}(\alpha), \quad \Phi_{\ell, r^{j}, m}(\alpha)
$$

with (37) are algebraically independent if $\alpha_{0,2} \neq 1$. If $\left(b_{h}\right)$ is not a constant sequence, then the same holds in the case $\alpha_{0,2}=1$. If $\left(b_{h}\right)$ is constant and $\alpha_{0,2}=1$, then the number $\Phi_{0,2,1}(\alpha)$ is algebraic but all other numbers in (41) are algebraically independent.

Theorem F3. Assume $r_{0}=4$, and let $\alpha_{\ell, r}, \beta_{\ell, r}$ satisfy the conditions of Lemma F1. Then the numbers

$$
\begin{array}{r}
\Gamma_{\mu, r^{j}}(\alpha), \quad X_{\ell, r^{j}, m}(\alpha), \quad Y_{\ell, r^{j}, m}(\alpha) \\
\left(r \in \mathcal{R}_{1}, 1 \leqslant \mu<r, 0<|\ell| \leqslant L, 1 \leqslant j \leqslant s, 1 \leqslant m \leqslant M\right)
\end{array}
$$

are algebraically independent. If $r_{0}=2$, then the same assertion holds for the numbers $\Gamma_{\mu, r^{j}}(\alpha), X_{\ell, r^{j}, m}(\alpha)$. 
The proofs of these results are similar, using the corresponding Lemmas F1-F4 above. Therefore, we give here only a proof for Theorem F1.

Proof of Theorem F1. We begin by applying Lemma 7 to the functions

$$
T_{\mu, r^{j}}\left(z_{1}, z_{2}\right), \quad U_{\ell, r^{j}, m}\left(z_{1}, z_{2}\right), \quad V_{\ell, r^{j}, m}\left(z_{1}, z_{2}\right)
$$

with $r, \mu, \ell, j, m$ as in (37). This gives a system of functional equations of the type used in [9, Corollary of Theorem 4]. Further, $\log r_{i}^{s ! p} / \log r_{k}^{s ! p} \notin \mathbb{Q}$ holds for distinct $r_{i}, r_{k} \in \mathcal{R}_{1}$. Therefore, by [9, Corollary of Theorem 4], to prove Theorem $\mathrm{F} 1$, it is enough to show that, for a given $r \in \mathcal{R}_{1}$, the functions

$$
\begin{aligned}
& T_{\mu, r^{j}}\left(z_{1}, z_{2}\right), \quad U_{\ell, r^{j}, m}\left(z_{1}, z_{2}\right), \quad V_{\ell, r^{j}, m}\left(z_{1}, z_{2}\right) \\
&(1 \leqslant \mu<r,-L \leqslant \ell \leqslant L, 1 \leqslant j \leqslant s, 1 \leqslant m \leqslant M)
\end{aligned}
$$

are algebraically independent over $\mathbb{C}\left(z_{1}, z_{2}\right)$. Thus, Lemma $\mathrm{F} 1$ implies the validity of the first part of Theorem F1. The proof of the second part is similar. We just replace [9, Corollary of Theorem 4] by [10, Lemma 2.1], and then use Lemma F2.

\section{Proof of Theorems 1-4}

We first introduce some preliminaries. By the definition of $R_{n}$ and $S_{n}$, we get

$$
\begin{array}{ll}
R_{k r^{h}+\ell}=D_{\ell, r} \gamma_{1}^{k r^{h}}\left(\left(\gamma_{2} / \gamma_{1}\right)^{k r^{h}}-d_{\ell, r}\right), & D_{\ell, r}=g_{2} \gamma_{2}^{\ell}, d_{\ell, r}=-g_{1} g_{2}^{-1}\left(\gamma_{1} / \gamma_{2}\right)^{\ell}, \\
S_{k r^{h}+\ell}=E_{\ell, r} \gamma_{1}^{k r^{h}}\left(\left(\gamma_{2} / \gamma_{1}\right)^{k r^{h}}-e_{\ell, r}\right), & E_{\ell, r}=h_{2} \gamma_{2}^{\ell}, e_{\ell, r}=-h_{1} h_{2}^{-1}\left(\gamma_{1} / \gamma_{2}\right)^{\ell} .
\end{array}
$$

Note here that $d_{\ell, r^{j}}, e_{\ell, r^{j}}, D_{\ell, r^{j}}$ and $E_{\ell, r^{j}}(j \in \mathbb{N})$ do not depend on $j$, and $\left|d_{\ell, r}\right|$ and $\left|e_{\ell, r}\right|$ do not depend on $r$. The condition $|\Omega| \notin\left|\gamma_{1} / \gamma_{2}\right|^{\mathbb{Z}}$ gives $\left|d_{\ell_{1}, r}\right| \neq\left|e_{\ell_{2}, r}\right|$ for $\ell_{1}, \ell_{2}$. Further, the condition $\Omega=\delta\left(\gamma_{1} / \gamma_{2}\right)^{\ell_{1}}$ means that $d_{\ell, r}=\delta e_{\ell_{1}+\ell, r}$ for all $\ell, r$. In particular, we then have $\left|d_{\ell, r}\right|=\left|e_{\ell_{1}+\ell, r}\right|$ for all $\ell, r$.

To prove Theorems $1-3$, we divide our consideration into three cases: i) $\gamma_{1}$ and $\gamma_{2}$ are multiplicatively independent, ii) $\gamma_{1} \gamma_{2}= \pm 1$, iii) $\gamma_{1}= \pm \gamma^{\kappa}, \gamma_{2}= \pm \gamma^{\nu}$ with $\kappa, \nu \in \mathbb{Z}$ satisfying $\kappa>\max (0, \nu)$. The cases i), ii) will lead to Theorems 1,2 , and case iii) to Theorem 3 .

Case $i$ ). Assume that the numbers (5) in Theorem 1 are algebraically dependent. Then there exist a finite set $\mathcal{R}_{0} \subset \mathbb{N} \backslash\{1\}$ and positive integers $L_{0}, M$ such that the numbers

$$
Q_{r}, R_{\ell, r, m}, S_{\ell, r, m} \quad\left(r \in \mathcal{R}_{0},-L_{0} \leqslant \ell \leqslant L_{0}, 1 \leqslant m \leqslant M\right)
$$

are algebraically dependent. Since we assumed $r \notin 2^{2 \mathbb{N}-1}$, there exist a finite set $\mathcal{R}_{1}$ with $4 \in \mathcal{R}_{1}$ as above and a positive integer $s$ such that $\mathcal{R}_{0} \subset\left\{r^{j}: r \in \mathcal{R}_{1}, 1 \leqslant\right.$ $j \leqslant s\}$. We choose $\alpha_{\ell, r}:=d_{\ell_{0}+\ell, r}, \beta_{\ell, r}:=e_{\ell_{1}+\ell_{0}+\ell, r}$ for $\ell \in \mathbb{Z}$ if some $\ell_{0}$ with 
$\left|d_{\ell_{0}, r}\right|=1$ exists; otherwise we choose $\ell_{0}=0$. Let $L=L_{0}+\left|\ell_{1}\right|+\left|\ell_{0}\right|$, and choose $h_{0}$ such that

$$
\left(\max \left(\left|\gamma_{1}\right|^{-k},\left|\gamma_{2} / \gamma_{1}\right|^{k}\right)\right)^{2^{h_{0}}}<\min \left(\left|\alpha_{-L, r}\right|,\left|\beta_{-L, r}\right|\right) .
$$

According to Theorem F1, the numbers

$$
\begin{aligned}
T_{1, r^{j}}(\alpha, \beta), \quad U_{\ell, r^{j}, m}(\alpha, \beta), \quad & V_{\ell, r^{j}, m}(\alpha, \beta) \\
& \left(r \in \mathcal{R}_{1},-L \leqslant \ell \leqslant L, 1 \leqslant j \leqslant s, 1 \leqslant m \leqslant M\right)
\end{aligned}
$$

are algebraically independent. Since, by (43) and (44),

$$
\begin{gathered}
Q_{r}-\sum_{h=0}^{h_{0}-1} \frac{a_{h}}{\gamma_{1}^{k r^{h}}}=T_{1, r}\left(\gamma_{1}^{-k},\left(\gamma_{2} / \gamma_{1}\right)^{k}\right), \\
R_{\ell, r, m}-\sum_{h=0}^{h_{0}-1} \frac{b_{h}}{\left(R_{k r^{h}+\ell}\right)^{m}}=D_{\ell, r}^{-m} U_{\ell-\ell_{0}, r, m}\left(\gamma_{1}^{-k},\left(\gamma_{2} / \gamma_{1}\right)^{k}\right), \\
S_{\ell, r, m}-\sum_{h=0}^{h_{0}-1} \frac{c_{h}}{\left(S_{k r^{h}+\ell}\right)^{m}}=E_{\ell, r}^{-m} V_{\ell-\ell_{1}-\ell_{0}, r, m}\left(\gamma_{1}^{-k},\left(\gamma_{2} / \gamma_{1}\right)^{k}\right),
\end{gathered}
$$

we have a contradiction to the assumption from the beginning of the proof. To show that the numbers (6) are algebraically independent, we just take $r_{0}=2$ in the above proof and use Theorem F2 (omitting the numbers $S_{\ell, r, m}$ ).

Case ii). In this case, $\gamma_{2}=\delta_{0} / \gamma_{1}, \delta_{0}= \pm 1$ holds, and the equations (43) and (44) now take the shape

$$
\begin{aligned}
& R_{k r^{h}+\ell}=D_{\ell, r} \gamma_{1}^{k r^{h}}\left(\gamma_{1}^{-2 k r^{h}}-d_{\ell, r}\right), \quad D_{\ell, r}=\delta_{0}^{k r} g_{2} \gamma_{2}^{\ell}, \quad d_{\ell, r}=-\delta_{0}^{k r+\ell} g_{1} g_{2}^{-1} \gamma_{1}^{2 \ell}, \\
& S_{k r^{h}+\ell}=E_{\ell, r} \gamma_{1}^{k r^{h}}\left(\gamma_{1}^{-2 k r^{h}}-e_{\ell, r}\right), \quad E_{\ell, r}=\delta_{0}^{k r} h_{2} \gamma_{2}^{\ell}, \quad e_{\ell, r}=-\delta_{0}^{k r+\ell} h_{1} h_{2}^{-1} \gamma_{1}^{2 \ell} \text {. }
\end{aligned}
$$

The condition $\Omega=\delta\left(\gamma_{1} / \gamma_{2}\right)^{\ell_{1}}=\delta \delta_{0}^{\ell_{1}} \gamma_{1}^{2 \ell_{1}}$ means that $d_{\ell, r}=\delta e_{\ell_{1}+\ell, r},\left|d_{\ell, r}\right|=$ $\left|e_{\ell_{1}+\ell, r}\right|$ for all $\ell, r$.

To prove the algebraic independence of the numbers (5), or of the numbers (6), we may now repeat the above proof in case i) and note that, in this case, (45)-(47) have the form

$$
\begin{gathered}
Q_{r}-\sum_{h=0}^{h_{0}-1} \frac{a_{h}}{\gamma_{1}^{k r^{h}}}=\Gamma_{1, r}\left(\gamma_{1}^{-k}\right), \\
R_{\ell, r, m}-\sum_{h=0}^{h_{0}-1} \frac{b_{h}}{\left(R_{k r^{h}+\ell}\right)^{m}}=D_{\ell, r}^{-m} \Phi_{\ell-\ell_{0}, r, m}\left(\gamma_{1}^{-k}\right), \\
S_{\ell, r, m}-\sum_{h=0}^{h_{0}-1} \frac{c_{h}}{\left(S_{k r^{h}+\ell}\right)^{m}}=E_{\ell, r}^{-m} \Lambda_{\ell-\ell_{1}-\ell_{0}, r, m}\left(\gamma_{1}^{-k}\right) .
\end{gathered}
$$


Furthermore, note that the condition $R_{\ell_{0}}=0$ is equivalent to $\alpha_{0,2}=d_{\ell_{0}, 2}=1$. Thus, Theorems 1 and 2 are proved.

Case iii). By denoting $\gamma_{1}=\delta_{1} \gamma^{\kappa}, \gamma_{2}=\delta_{2} \gamma^{\nu}$, we now have

$$
\begin{aligned}
R_{k r^{h}+\ell} & =D_{\ell, r} \gamma^{k \kappa r^{h}}\left(\gamma^{k(\nu-\kappa) r^{h}}-d_{\ell, r}\right), & D_{\ell, r} & =\delta_{2}^{k r+\ell} g_{2} \gamma^{\nu \ell}, \\
d_{\ell, r} & =-\left(\delta_{1} \delta_{2}\right)^{k r+\ell} g_{1} g_{2}^{-1} \gamma^{(\kappa-\nu) \ell}, & S_{k r^{h}+\ell} & =E_{\ell, r} \gamma^{k \kappa r^{h}}\left(\gamma^{k(\nu-\kappa) r^{h}}-e_{\ell, r}\right), \\
E_{\ell, r} & =\delta_{2}^{k r+\ell} h_{2} \gamma^{\nu \ell}, & e_{\ell, r} & =-\left(\delta_{1} \delta_{2}\right)^{k r+\ell} h_{1} h_{2}^{-1} \gamma^{(\kappa-\nu) \ell} .
\end{aligned}
$$

In this case, the condition $\Omega=\delta\left(\gamma_{1} / \gamma_{2}\right)^{\ell_{1}}=\delta\left(\delta_{1} \delta_{2}\right)^{\ell_{1}} \gamma^{(\kappa-\nu) \ell_{1}}$ means once more that $d_{\ell, r}=\delta e_{\ell_{1}+\ell, r},\left|d_{\ell, r}\right|=\left|e_{\ell_{1}+\ell, r}\right|$ for all $\ell, r$. Note also that $\left|d_{\ell_{0}, r}\right|=1$ if and only if $\left|g_{1} g_{2}^{-1}\left(\gamma_{1} / \gamma_{2}\right)^{\ell_{0}}\right|=1$.

Again, as in case i) above,

$$
\begin{gathered}
Q_{r}^{*}-\sum_{h=0}^{h_{0}-1} \frac{a_{h}}{\gamma^{k r^{h}}}=\Gamma_{1, r}\left(\gamma^{-k}\right), \\
R_{\ell, r, m}-\sum_{h=0}^{h_{0}-1} \frac{b_{h}}{\left(R_{k r^{h}+\ell}\right)^{m}}=D_{\ell, r}^{-m} X_{\ell-\ell_{0}, r, m}\left(\gamma^{-k}\right), \\
S_{\ell, r, m}-\sum_{h=0}^{h_{0}-1} \frac{c_{h}}{\left(S_{k r^{h}+\ell}\right)^{m}}=E_{\ell, r}^{-m} Y_{\ell-\ell_{1}-\ell_{0}, r, m}\left(\gamma^{-k}\right) .
\end{gathered}
$$

The validity of Theorem 3 now follows from Theorem F3.

Acknowledgement. The authors are grateful to the referee for suggestions improving the readability of the work.

\section{References}

[1] P.-G. Becker, T. Töpfer, Transcendency results for sums of reciprocals of linear recurrences, Math. Nachr. 168 (1994) 5-17.

[2] P. Bundschuh, K. Väänänen, Some arithmetical results on reciprocal sums of certain Fibonacci-type numbers, Southeast Asian Bull. Math. (to appear).

[3] P. Bundschuh, K. Väänänen, Algebraic independence of certain Mahler functions and of their values, J. Aust. Math. Soc. 98 (2015) 289-310.

[4] P. Bundschuh, K. Väänänen, Algebraic independence of reciprocal sums of certain Fibonacci-type numbers, arXiv:1403.5510v1 [math. NT] (2014).

[5] D. Duverney, T. Kanoko, T. Tanaka, Transcendence of certain reciprocal sums of linear recurrences, Monatsh. Math. 137 (2002) 115-128.

[6] T. Kanoko, T. Kurosawa, I. Shiokawa, Transcendence of reciprocal sums of binary recurrences, Monatsh. Math. 157 (2009) 323-334.

[7] K. Nishioka, Mahler Functions and Transcendence, LNM 1631 (Springer, Berlin et al., 1996). 
[8] K. Nishioka, Algebraic independence of reciprocal sums of binary recurrences, Monatsh. Math. 123 (1997) 135-148.

[9] K. Nishioka, Algebraic independence of reciprocal sums of binary recurrences II, Monatsh. Math. 136 (2002) 123-141.

[10] K. Nishioka, T. Tanaka, T. Toshimitsu, Algebraic independence of sums of reciprocals of the Fibonacci numbers, Math. Nachr. 202 (1999) 97-108.

Addresses: Peter Bundschuh: Mathematisches Institut, Universität zu Köln, Weyertal 86-90, 50931 Köln, Germany;

Keijo Väänänen: Department of Mathematical Sciences, University of Oulu, P. O. Box 3000, 90014 Oulu, Finland.

E-mail: pb@math.uni-koeln.de, keijo.vaananen@oulu.fi

Received: 28 October 2014; revised: 29 April 2015 